\title{
Clinical and microbiological characteristics of Klebsiella pneumoniae liver abscess in East China
}

Ting-ting Qu ${ }^{1,4}$, Jian-cang Zhou ${ }^{2}$, Yan Jiang ${ }^{2}$, Ke-ren Shi ${ }^{2}$, Bin $\mathrm{Li}^{3}$, Ping Shen ${ }^{1}$, Ze-qing Wei ${ }^{1}$ and Yun-song Yu ${ }^{1,2^{*}}$

\begin{abstract}
Background: Klebsiella pneumoniae has been the dominant pathogen for liver abscesses in several Asian countries. Although the prevalence of $K$. pneumoniae liver abscess (KLA) in mainland China is increasing recently, the clinical and microbiological characteristics of KLA in China have not been elucidated.

Methods: Clinical and microbiology characteristics of 45 consecutive patients with KLA from a tertiary teaching hospital in China between June 2008 and June 2012 were retrospectively evaluated.

Results: Vast majority of the strains were susceptible to main antimicrobial agents. Most of K. pneumoniae strains from pyogenic liver abscess patients belonged to K1/K2 serotype (68.9\% for K1 serotype and 20\% for K2 serotype). All K. pneumoniae strains were rmpA positive, and $68.9 \%$ of these strains were magA positive. Overall, 57.8\% (26/45) of K. pneumoniae strains belonged to ST23. Twenty-five of 26 ST23 K. pneumoniae isolates (96.2\%) from KLA patients were magA-positive and K1 serotype. Only 28.9\% (13/45) of KLA isolates exhibited hypermucoviscous phenotype, which is clinically used as the characteristic of hypervirulent $K$. pneumoniae (hvKP). Liver abscess sizes in patients infected with hvKP were tend to be larger than those in patients infected with cKP. There was no significant association between the microbiological and clinical characteristics including serotypes, magA and rmpA genotypes, and STs with the metastatic infection and prognosis of KLA.
\end{abstract}

Conclusions: Neither the serotypes, magA and rmpA genotypes, nor the STs of K. pneumoniae were associated with the metastatic infection and prognosis of KLA. However, further studies with larger sample are needed in the future.

Keywords: Klebsiella pneumoniae, K. pneumoniae liver abscess (KLA), Hypermucoviscous, magA, Multilocus sequence typing (MLST)

\section{Background}

Klebsiella pneumoniae has emerged as the dominant cause of pyogenic liver abscess in Asia and then was found worldwide. This condition is frequently associated with severe complications, including septic endophthalmitis and other extrahepatic lesions infections, especially in patients with diabetes [1-3]. This new $K$. pneumoniae variant was defined as hypervirulent $K$. pneumonia (hvKP). Furthermore, the ability for metastatic spread of infection demonstrated by hvKP is different from classic K. pneumoniae (cKP). Given there is no clear marker for

\footnotetext{
*Correspondence: yvys119@163.com

'State Key Laboratory for Diagnosis and treatment of Infectious Disease, First Affiliated Hospital, College of Medicine, Zhejiang University, 3\# Qingchun East Road, Hangzhou 310016, China

2Department of Infectious Diseases, Sir Run Run Shaw Hospital, College of Medicine, Zhejiang University, Hangzhou, Zhejiang, China

Full list of author information is available at the end of the article
}

hvKP strains, a hypermucoviscous phenotype has been considered to be associated with strains that cause KLA. This mucoid phenotype might be indicative of the extent of capsular polysaccharide expression, which is related to resistance to phagocytosis. Currently, hepato-virulent K. pneumoniae causing primary hepatic abscesses has posed a challenge for early laboratory identification and recognition. Bacteraemic K. pneumoniae isolates with positive string tests should be considered invasive strains capable of causing disseminated infection because the increased virulence associated with hyper virulent $(\mathrm{HV})$ strains $[4,5]$.

Capsular serotypes, magA, and rmpA have been documented in high prevalence for $K$. pneumonia liver abscess. Several studies of bacterial pathogenesis in Taiwan have documented that serotype $\mathrm{K} 1$ or $\mathrm{K} 2$, magA, and rmpA are possible virulence factors in $K$. pneumoniae 
liver abscess. The magA gene actually corresponds to the capsular polysaccharide synthesis (cps) gene wzy of $K$. pneumoniae isolates of serotype K1 [6-10]. The $\operatorname{rmp} A$ gene is a plasmid-mediated regulator of extracellular polysaccharide synthesis, and $r m p A$-carrying strains were associated with the hypermucoviscosity phenotype $[8,11,12]$.

Multilocus Sequence typing (MLST) is an increasingly common used molecular epidemiologic approach for categorizing strains, and a typing screen has been described for $K$. pneumoniae. ST23 was most commonly described so far and is strongly associated with the K1 capsular serotype and liver abscess [2,13]. However, ST-65-like and -86-like are the two major MLST types among serotype K2 isolates from Asia [2,14].

In mainland China, the prevalence of $K$. pneumoniae liver abscess (KLA) is high, while only some KLA cases were reported in the English language scientific literature. Recently, Luo et al. firstly reported the molecular epidemiology and prevalence of virulence factors of the 51 KLA isolates in a Chinese hospital. However, very little clinical information was collected and evaluated in their study [15]. In our study, we analyzed clinical and microbiological characteristics of 45 KLA from a medical center in China between June 2008 and June 2012. To investigate the relationship of the clinical and microbiology characteristics of KLA, the present study evaluated the clinical manifestations of patients and detected the antimicrobial agents susceptibility, K1/K2 serotypes, MLST, hypermucoviscous phenotype, and magA/rmpA genotypes of the related $K$. pneumoniae isolates from KLA patients in a medical center over a 4-year period in East China.

\section{Methods}

\section{Study design and setting}

The study was a retrospective review of all patients with KLA in the First Affiliated Hospital, College of Medicine, Zhejiang University, an urban, 2500-bed major tertiary teaching hospital in Hangzhou, East China, from June 1, 2008 to June 30, 2012. The institutional review board of the First Affiliated Hospital, College of Medicine, Zhejiang University approved the study protocol and waived from the need for a consent form.

The hospital was one of the leading hospitals for liver transplantation in China and has an approximate annual admission of 108,700 . It has a quaternary referral hepato-biliary unit and therefore, many patients with hepato-biliary diseases were transferred from rural hospitals to our hospital. In 2013, it became the one of the public hospitals in mainland China to be accredited by the Joint Commission International, a US-based, World Health Organization-authorized organization for medical quality evaluation.

\section{Data sources and definition of KLA and outcomes}

The medical records were retrospectively reviewed to extract all the patients with a diagnosis of KLA during the study period. A diagnosis of a KLA was defined with the combination of presence of the typical clinical manifestations of infection, such as fever, sepsis and right upper abdominal pain, imaging evidence, and positive aspiration that was consistent with a pyogenic liver abscess (PLA). The patients with KLA who were included in our study met the following criteria: 1) a PLA was the primary cause of the hospitalization but not a complication; 2) older than 18 years old; 3) PLA patients with $K$. pneumoniae culture-positive of drained abscess. Thus, PLA patients without drainage but with positive blood cultures of K. pneumoniae were excluded in this study. As practiced in our hospital, lab examinations for all patients with KLA are required on the admission and generally every $2-3$ days at the discretion of the attending physicians.

For each enrolled patient, the following data elements were extracted: 1) demographic characteristics (age, gender); (2) coexisting conditions; (3) location and size of abscess; (4) laboratory examinations; (5) hospital outcomes. Primary outcome was hospital mortality, and secondary outcome were complications including septic shock and metastatic infections to extra-hepatic sites, such as spontaneous bacterial peritonitis, pneumonia, endophthalmitis.

\section{Antimicrobial susceptibility testing}

Susceptibility testing for the $45 \mathrm{~K}$. pneumoniae strains was performed using the E-test strip according to the manufacturer's instructions. Results were interpreted according to the recommendations and definitions from Clinical and Laboratory Standards Institute (CLSI). Antimicrobial agents tested included amikacin, amoxicillinclavulanate, ampicillin, ampicillin-sulbactam, aztreonam, cefoxitin, cefuroxime, cefepime, cefotaxime, ceftazidime, ciprofloxacin, gentamicin, piperacillin, piperacillintazobactam, cefoperazone-sulbactam, tetracycline, minocycline, sulphamethoxazole-trimethoprim (SMZ-TMP), imipenem and meropenem.

\section{Extended-spectrum beta-lactamases (ESBLs) detection}

The combination-disk synergy tests using cefotaxime $(30 \mu \mathrm{g}) \pm$ clavulanic acid $(10 \mu \mathrm{g})$ and ceftazidime $(30 \mu \mathrm{g}) \pm$ clavulanic acid $(10 \mu \mathrm{g})$ were performed to detect ESBLs phenotype for all the collected isolates. The ESBLs phenotype was confirmed by $5-\mathrm{mm}$ or greatly increased zone diameter for either cefotaxime or ceftazidime in combination with clavulanic acid versus its zone when tested alone.

\section{String test}

Strains with the hypermucoviscosity phenotype were defined as high virulent. A string test was performed to 
distinguish hvKP from cKP. A positive string test was defined as the formation of a viscous string $>5 \mathrm{~mm}$ in length when bacterial colonies on an agar plate are stretched by an inoculation loop [5].

\section{PCR-mediated detection of rmpA and capsular serotype-specific genes}

Genomic DNA was extracted from all $K$. pneumoniae strains (QIAGEN DNA extraction kit, QIAGEN, USA) and the rmpA and serotype-specific genes for the K1, and $\mathrm{K} 2$ capsular serotypes were amplified by polymerase chain reaction (PCR) as previously reported $[16,17]$.

\section{Multilocus sequence typing (MLST) and Pulsed field gel electrophoresis (PFGE)}

MLST of seven housekeeping genes (gapA, $m d h$, phoE, $\operatorname{ton} B$, infB, pgi, and rpoB) was performed according to the protocol described on the $K$. pneumoniae MLST website (http://bigsdb.web.pasteur.fr/klebsiella/primers_used.html). Alleles and sequence types (STs) were assigned by using the MLST database (http://www.pasteur.fr/cgi-bin/genopole/PF8/mlstdbnet.pl?file=klebs_profiles.xml). Alleles and STs that had not been described previously were submitted to the database.

Total DNA was prepared and PFGE was performed as described previously [18]. The PFGE dendrogram was created by Software BioNumerics 6.6. The results were interpreted according to the criteria suggested by Tenoval et al. [19].

\section{Statistical analysis}

Statistical analysis was performed using SPSS 16.0 (Chicago, Ill, USA). Descriptive data were reported as either mean \pm SD or number and percentage. Normally distribution of the data was tested with KolmogorovSmirnov test. With respect to the differences in outcomes between various groups, categorical variables were compared using chi-square analysis. Where the number of cases was smaller than 5, the Fisher's exact test was used. Continuous variables were compared using Independent Sample T test for normally distributed data and MannWhitney $U$ test for non-normally distributed data. Significance was defined as a $P$ value $<0.05$.

\section{Results}

\section{Patients' characteristics}

From June 2008 to June 2012, a total of 45 liver abscess patients admitted to our hospital were diagnosed as KLA with the $K$. pneumoniae culture-positive of their liver abscess drainage. Demographic data and clinical characteristics were shown in Table 1.

Twenty-seven (60.0\%) were males and 18 (40.0\%) were females. The mean age was $53.7 \pm 11.2$ years. Diabetes mellitus was found in 22 patients (48.9\%), followed by
Table 1 Clinical characteristics of $K$. pneumoniae liver abscess in China

\begin{tabular}{|c|c|}
\hline Clinical characteristic & Value (n, \%) \\
\hline \multicolumn{2}{|l|}{ Gender } \\
\hline Male & $27(60.0 \%)$ \\
\hline Female & $18(40.0 \%)$ \\
\hline \multicolumn{2}{|l|}{ Age (yr) } \\
\hline mean age & $53.7 \pm 11.2(18-73)$ \\
\hline $18-49$ & 14(31.1\%) \\
\hline $50-64$ & $23(51.1 \%)$ \\
\hline$\geq 65$ & $8(17.8 \%)$ \\
\hline \multicolumn{2}{|l|}{ Coexisting condition } \\
\hline Diabetes mellitus & $22(48.9 \%)$ \\
\hline Biliary tract diseases & $16(35.6 \%)$ \\
\hline Abdominal surgery & $10(22.2 \%)$ \\
\hline Immunosuppression* & 0 \\
\hline No underlying diseases & $5(11.1 \%)$ \\
\hline Current drinker & $14(31.1 \%)$ \\
\hline \multicolumn{2}{|l|}{ Abscess } \\
\hline Single & $40(88.9 \%)$ \\
\hline Multiple & $5(11.1 \%)$ \\
\hline Right hepatic lobe & $35(77.9 \%)$ \\
\hline Left hepatic lobe & $7(15.6 \%)$ \\
\hline Both lobes & $3(6.7 \%)$ \\
\hline \multicolumn{2}{|l|}{ Abscess size $(\mathrm{cm})$} \\
\hline$<5$ & $5(11.1 \%)$ \\
\hline $5-10$ & $33(73.3 \%)$ \\
\hline$>10$ & $7(15.6 \%)$ \\
\hline \multicolumn{2}{|l|}{ Complications } \\
\hline Metastatic infections & 14(31.1\%) \\
\hline Spontaneous bacterial peritonitis & $3(6.7 \%)$ \\
\hline Pneumonia & $10(22.2 \%)$ \\
\hline Endophthalmitis & $1(2.2 \%)$ \\
\hline Septic shock & $3(6.7 \%)$ \\
\hline \multicolumn{2}{|l|}{ Lab examination } \\
\hline WBC $\left(\times 10^{9} / \mathrm{L}\right)$ & $11.9(7.0-28.1)$ \\
\hline CRP (mg/L) & $106.9(28.8-311.0)$ \\
\hline Mortality & 0 \\
\hline
\end{tabular}

*Immunosuppression may have been caused by the presence of human immunodeficiency virus infection, chemotherapy or radiotherapy within 1 month before the onset of illness, or glucocorticoid therapy (equivalent of $30 \mathrm{mg}$ of prednisone per day) for 15 days before the onset of illness.

biliary tract disease found in 16 patients (35.6\%). Ten patients $(22.2 \%)$ had a history of abdominal surgery. No patient was in immunosuppression condition (definition shown below Table 1), and 5 patients (11.1\%) had no underlying diseases. 
Forty patients had solitary abscess and 5 patients (11.1\%) had multiple abscesses. Among them, 35 (77.9\%) had abscesses in the right hepatic lobe, 7 (15.6\%) in the left hepatic lobe, and $3(6.7 \%)$ in both hepatic lobes. Fourteen patients (31.1\%) had metastatic infections, including 3 patients with spontaneous bacterial peritonitis, 10 with pneumonia and 1 with endophthalmitis. Three patients had septic shock. Overall, none of the patients died.

Leukocytosis was found in patients with a median WBC count of $11.9 \times 10^{9} / \mathrm{L}\left(7.0-28.1 \times 10^{9} / \mathrm{L}\right)$, and CRP in patients had a median count of $106.9 \mathrm{mg} / \mathrm{L}(28.8$ $311.0 \mathrm{mg} / \mathrm{L}$ ).

\section{Microbiologic characterization of the K. pneumoniae strains}

Only 1 of $45 K$. pneumoniae strains was ESBL-positive. All the isolates were resistant to ampicillin, but eleven were unsusceptible to piperacillin, two to cefuroxime, one to cefotaxime, one to ciprofloxacin, one to gentamicin, and one to cefepime (Table 2). All the isolates were susceptible to amikacin, amoxicillin-clavulanate, aztreonam, ceftazidime, cefoperazone-sulbactam, SMZ-TMP, imipenem, and meropenem, as shown in Table 2.

MLST analysis revealed a total of 15 genotypes among the $45 \mathrm{~K}$. pneumoniae strains. With respect to the sequence types (ST), more than half were ST23 ( $\mathrm{n}=26$, $57.8 \%$ ), followed by ST65 and ST86 (both $n=3$ ), ST375 $(\mathrm{n}=2)$, and ST25, ST29, ST30, ST163, ST367, ST374, ST380, ST660, ST700, ST806, and ST1049 ( $\mathrm{n}=1$ for all), as shown in Additional file 1: Table S1. The PFGE results showed that the 26 ST23 K. pneumoniae strains had 12 separated pulsetypes, as shown in Figure 1.

Among $45 \mathrm{~K}$. pneumoniae strains, $\mathrm{K} 1$ was the dominant serotype (31 strains, $68.9 \%$ ), followed by K2 serotype (9 strains, 20\%) and non K1/K2 serotype (5 strains, 11.1\%). As shown in Additional file 1: Table S1, 31 serotype K1 KLA K. pneumoniae isolates mainly belonged to ST23-like (83.9\%), while STs of these 9 serotype K2 isolates were ST65-like (5 strains), ST86-like (2 strains), ST23-like (1 strain), and ST374 (1 strain).

Virulence genes analysis showed 31 strains (68.9\%) were magA gene positive and all 45 strains were $\operatorname{rmp} A$ gene positive, as shown in Table 2. According to the string test results, $13 \mathrm{hvKP}$ isolates were obtained at the rate of $28.9 \%$. All of the $13 \mathrm{hvKP}$ strains belonged to $\mathrm{K} 1 / \mathrm{K} 2$ serotype (7 K1 isolates and $6 \mathrm{~K} 2$ isolates).

\section{Comparison of microbiological characteristics between ST23 and non-ST23 K. pneumoniae isolates}

As shown in Table 3, the prevalence of $\mathrm{K} 1$ serotype or magA-positive isolates in ST23 K. pneumoniae isolates was significantly higher than that in non-ST23 isolates (96.2\% vs 31.6\%, $P<0.001$ ). Conversely, the prevalence of K2 serotype in ST23 K. pneumoniae isolates was
Table 2 Microbiological characteristics of $K$. pneumoniae liver abscess

\begin{tabular}{|c|c|}
\hline Microbiological characteristic & Value $(n, \%)$ \\
\hline \multicolumn{2}{|l|}{ Serotypes } \\
\hline K1 & $31(68.9 \%)$ \\
\hline K2 & $9(20 \%)$ \\
\hline Non K1/K2 & $5(11.1 \%)$ \\
\hline \multicolumn{2}{|l|}{ Virulence genes } \\
\hline magA+ & $31(68.9 \%)$ \\
\hline rmpA+ & $45(100.0 \%)$ \\
\hline \multicolumn{2}{|l|}{ MLST } \\
\hline ST23 & $26(57.8 \%)$ \\
\hline Non-ST23 & $19(42.2 \%)$ \\
\hline hvKP & $13(28.9 \%)$ \\
\hline CKP & $32(71.1 \%)$ \\
\hline ESBLs positive & $1(2.2 \%)$ \\
\hline \multicolumn{2}{|l|}{ Antimicrobial susceptibility (S\%) } \\
\hline Amikacin & $100 \%$ \\
\hline Amoxicillin-clavulanate & $100 \%$ \\
\hline Ampicillin & $0 \%$ \\
\hline Ampicillin-sulbactam & $93.5 \%$ \\
\hline Aztreonam & $100 \%$ \\
\hline Cefoxitin & $95.7 \%$ \\
\hline Cefuroxime & $95.7 \%$ \\
\hline Cefepime & $97.8 \%$ \\
\hline Cefotaxime & $97.8 \%$ \\
\hline Ceftazidime & $100 \%$ \\
\hline Ciprofloxacin & $97.8 \%$ \\
\hline Gentamicin ,CN10 & $97.8 \%$ \\
\hline Piperacillin & $76.1 \%$ \\
\hline Piperacillin-tazobactam & $97.8 \%$ \\
\hline Cefoperazone-sulbactam & $100 \%$ \\
\hline Tetracycline & $93.5 \%$ \\
\hline Minocycline & $93.5 \%$ \\
\hline SMZ-TMP & $100 \%$ \\
\hline Imipenem & $100 \%$ \\
\hline Meropenem & $100 \%$ \\
\hline
\end{tabular}

significantly lower than that in non-ST23 isolates $(0 \%$ vs 47.4\%, $P<0.001)$.

\section{Comparison of microbiological characteristics between hvKP and CKP isolates from KLA}

As shown in Table 4, the prevalence of K2 serotype in hvKP isolates was significantly higher than that in cKP isolates $(46.2 \%$ vs 9.4\%, $P=0.011)$. All of the 5 non-K1/K2 serotype isolates belonged to cKP, including 1 ST23 and 4 non-ST23 isolates. No significant 


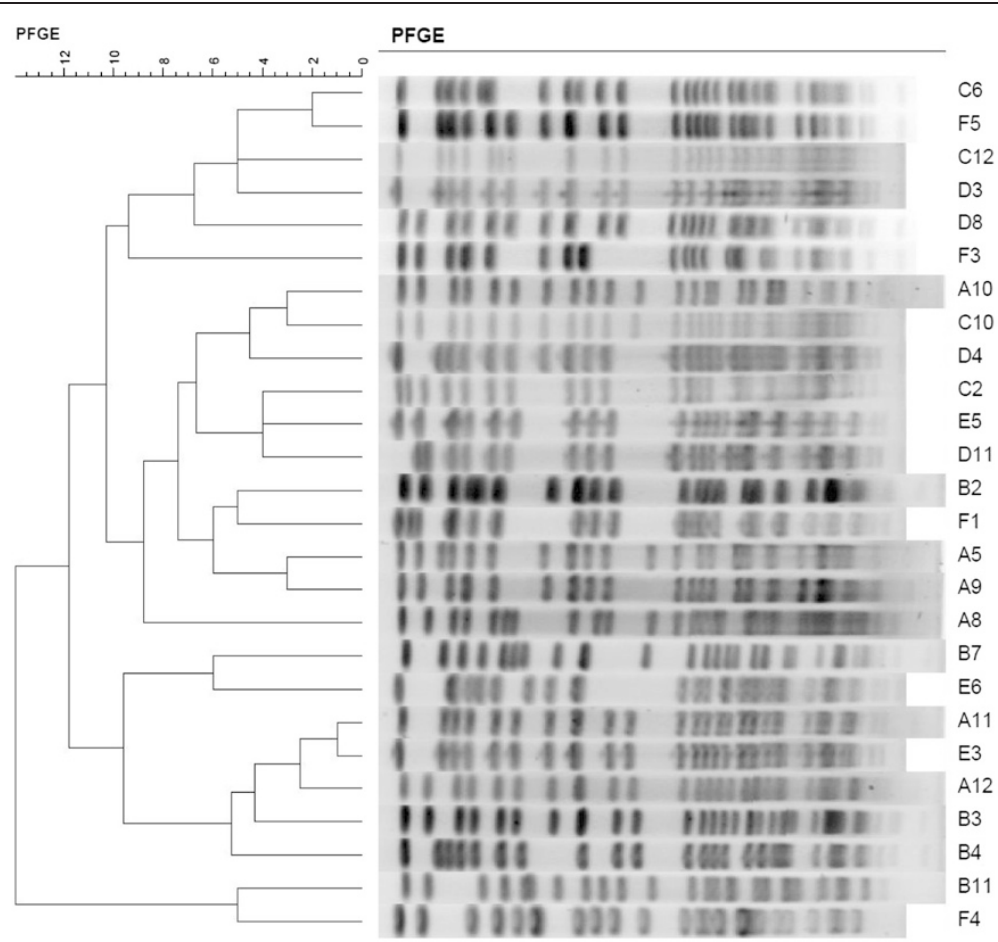

Figure 1 PFGE analysis of the 26 ST23 K. pneumoniae strains.

difference was found between the magA positive rates in hvKP and cKP strains (53.8\% vs 75\%).

Comparison of clinical characteristics of KLA patients infected with different $K$. pneumoniae isolates Comparison of clinical characteristics between KLA patients infected with magA-negative and -positive K. pneumoniae isolates

As shown in Table 5, there is a trend that more patients with biliary tract diseases in KLA patients infected with

Table 3 Comparison of microbiological characteristics between ST23 and non-ST23 K. pneumoniae isolates from KLA

\begin{tabular}{llll}
\hline $\begin{array}{l}\text { Microbiological } \\
\text { characteristic }\end{array}$ & $\begin{array}{l}\text { ST23 }(\mathbf{n}=\mathbf{2 6}) \\
\text { No. (\%) }\end{array}$ & $\begin{array}{l}\text { Non-ST23 }(\mathbf{n}=19) \\
\text { No. (\%) }\end{array}$ & $\begin{array}{l}\boldsymbol{P} \text {-value } \\
\text { Serotypes -no. (\%) }\end{array}$ \\
K1 & $25(96.2 \%)$ & $6(31.6 \%)$ & $<0.001$ \\
K2 & $0(0.0 \%)$ & $9(47.4 \%)$ & $<0.001$ \\
Non K1/K2 & $1(3.8 \%)$ & $4(21.1 \%)$ & 0.146 \\
Virulence genes -no. (\%) & & & \\
magA+ & $25(96.2 \%)$ & $6(31.6 \%)$ & $<0.001$ \\
rmpA+ & $26(100.0 \%)$ & $19(100 \%)$ & 1 \\
hvKP -no. (\%) & $6(23.1 \%)$ & $7(36.8 \%)$ & 0.341 \\
CKP -no. (\%) & $20(76.9 \%)$ & $12(63.2 \%)$ & 0.341 \\
ESBLs positive -no. (\%) & $1(3.8 \%)$ & $0(0.0 \%)$ & 0.387 \\
\hline
\end{tabular}

magA-negative $K$. pneumoniae isolates than those in patients with magA-positive $K$. pneumoniae isolates (57.1\% vs $25.8 \%, P=0.053)$. There were no significant differences of liver abscess sizes and complications between magApositive and -negative K. pneumoniae isolates.

\section{Comparison of clinical characteristics between KLA patients infected with hvKP and cKP isolates}

More patients infected with cKP tend to have a history of diabetes mellitus or drinking than that of hvKP patients $(56.3 \%$ vs $30.8 \%$ and $37.5 \%$ vs $15.4 \%$ respectively,

Table 4 Comparison of microbiological characteristics between hvKP and CKP isolates from KLA

\begin{tabular}{llll}
\hline $\begin{array}{l}\text { Microbiological } \\
\text { characteristic }\end{array}$ & $\begin{array}{l}\text { hvKP }(\mathbf{n}=13) \\
\text { No. (\%) }\end{array}$ & $\begin{array}{l}\text { cKP }(\mathbf{n}=\mathbf{3 2}) \\
\text { No. (\%) }\end{array}$ & $\begin{array}{l}P \text {-value } \\
\text { Serotypes -no. (\%) }\end{array}$ \\
K1 & $7(53.8 \%)$ & $24(75 \%)$ & 0.286 \\
K2 & $6(46.2 \%)$ & $3(9.4 \%)$ & 0.011 \\
Non K1/K2 & $0(0.0 \%)$ & $5(15.6 \%)$ & 0.301 \\
Virulence genes -no. (\%) & & & \\
magA+ & $7(53.8 \%)$ & $24(75 \%)$ & 0.286 \\
rmpA+ & $13(100 \%)$ & $32(100 \%)$ & \\
ST23-no. (\%) & $6(46.2 \%)$ & $20(62.5 \%)$ & 0.341 \\
Non-ST23 -no. (\%) & $7(53.8 \%)$ & $12(37.5 \%)$ & 0.341 \\
ESBLs positive -no. (\%) & $1(7.7 \%)$ & $0(0.0 \%)$ & 0.219 \\
\hline
\end{tabular}


Table 5 Comparison of clinical characteristics of KLA patients infected with different K. pneumoniae isolates

\begin{tabular}{|c|c|c|c|c|c|c|c|c|c|}
\hline \multirow[t]{3}{*}{ Clinical characteristic } & \multicolumn{3}{|c|}{ ST23 and non-ST23 } & \multicolumn{3}{|c|}{ hvKP and CKP } & \multicolumn{3}{|c|}{ magA-positive and -negative } \\
\hline & \multirow{2}{*}{$\begin{array}{l}\text { ST23 } \\
(n=26) \\
\text { No. }(\%)\end{array}$} & \multirow{2}{*}{$\begin{array}{l}\text { Non-ST23 } \\
(n=19) \\
\text { No. }(\%)\end{array}$} & \multirow[t]{2}{*}{$\mathbf{P}$} & \multirow{2}{*}{$\begin{array}{l}\text { hvKP } \\
(n=13) \\
\text { No. }(\%)\end{array}$} & \multirow{2}{*}{$\begin{array}{l}\text { cKP } \\
(n=32) \\
\text { No. }(\%)\end{array}$} & \multirow[t]{2}{*}{$P$} & \multirow{2}{*}{$\begin{array}{l}\text { magA positive } \\
(n=31) \\
\text { No. }(\%)\end{array}$} & \multirow{2}{*}{$\begin{array}{l}\text { magA negative } \\
(\mathrm{n}=14) \\
\text { No. }(\%)\end{array}$} & \multirow[t]{2}{*}{$\mathbf{P}$} \\
\hline & & & & & & & & & \\
\hline Male sex & $17(65.4 \%)$ & $11(57.9 \%)$ & 0.757 & $8(61.5 \%)$ & 18(56.3\%) & 1 & $17(54.8 \%)$ & $9(64.3 \%)$ & 0.746 \\
\hline Age & $54.8 \pm 11.9$ & $52.3 \pm 10.4$ & 0.465 & $52.3 \pm 8.7$ & $54.3 \pm 12.2$ & 0.600 & $53.9 \pm 12.5$ & $53.2 \pm 8.2$ & 0.844 \\
\hline \multicolumn{10}{|l|}{ Coexisting condition } \\
\hline Diabetes mellitus & 13(50.0\%) & $9(47.4 \%)$ & 1 & $4(30.8 \%)$ & 18(56.3\%) & 0.189 & $16(50 \%)$ & $6(42.9 \%)$ & 0.749 \\
\hline Biliary tract diseases & $8(30.8 \%)$ & $8(17.8 \%)$ & 0.534 & $4(30.8 \%)$ & $12(37.5 \%)$ & 0.743 & $8(25.8 \%)$ & $8(57.1 \%)$ & 0.053 \\
\hline Abdominal surgery & $6(23.1 \%)$ & $4(21.1 \%)$ & 1 & $3(23.1 \%)$ & $7(21.9 \%)$ & 1 & $7(22.6 \%)$ & $3(21.4 \%)$ & 1 \\
\hline No underlying diseases & $3(11.5 \%)$ & $2(5.3 \%)$ & 1 & $3(23.1 \%)$ & $2(6.3 \%)$ & 0.136 & $3(9.7 \%)$ & $2(14.3 \%)$ & 0.639 \\
\hline Current drinker & $10(38.5 \%)$ & $4(21.1 \%)$ & 0.330 & $2(15.4 \%)$ & $12(37.5 \%)$ & 0.178 & $11(35.5 \%)$ & $3(21.4 \%)$ & 0.492 \\
\hline Abscess size (diameter, cm) & $7.7 \pm 2.4$ & $7.4 \pm 2.8$ & 0.691 & $8.8 \pm 2.4$ & $7.1 \pm 2.5$ & 0.057 & $7.5 \pm 2.3$ & $7.9 \pm 3.0$ & 0.605 \\
\hline \multicolumn{10}{|l|}{ Complications } \\
\hline Metastatic infections & $10(38.5 \%)$ & $4(21.1 \%)$ & 0.330 & $5(38.5 \%)$ & $9(28.1 \%)$ & 0.486 & $11(35.5 \%)$ & $3(21.4 \%)$ & 0.492 \\
\hline Spontaneous bacterial peritonitis & $3(11.5 \%)$ & $0(0.0 \%)$ & 1 & $0(0.0 \%)$ & $3(9.4 \%)$ & 0.546 & $3(9.7 \%)$ & $0(0.0 \%)$ & 0.541 \\
\hline Pneumonia & $6(23.1 \%)$ & $4(21.1 \%)$ & 1 & $3(23.1 \%)$ & $7(21.9 \%)$ & 1 & $7(22.6 \%)$ & $3(21.4 \%)$ & 1 \\
\hline Endophthalmitis & $1(3.8 \%)$ & $0(0.0 \%)$ & 1 & $0(0.0 \%)$ & $1(3.1 \%)$ & 1 & $1(3.2 \%)$ & $0(0.0 \%)$ & 1 \\
\hline Septic shock & $3(11.5 \%)$ & $0(0.0 \%)$ & 0.258 & $2(15.4 \%)$ & $1(3.1 \%)$ & 0.196 & $3(9.7 \%)$ & $0(0.0 \%)$ & 0.541 \\
\hline
\end{tabular}

$P=0.189$ and 0.178 , respectively). There were 3 hvKP $(3 / 13,23.1 \%)$ patients and 2 cKP $(2 / 32,6.3 \%)$ without any underlying diseases. Liver abscess sizes in patients infected with hvKP $(8.8 \pm 2.4 \mathrm{~cm})$ were tend to be larger than those in patients infected with $\mathrm{CKP}(7.1 \pm 2.5 \mathrm{~cm})$ $(P=0.057)$ (Table 5).

Comparison of clinical characteristics between KLA patients infected with ST23 and non-ST23 K. pneumoniae isolates

Among 26 patients infected with ST23 K. pneumoniae strains, 21 patients $(80.8 \%)$ had solitary abscess and 5 patients (19.2\%) had multiple abscesses. However, all of 19 patients $(100 \%)$ infected with non-ST23 K. pneumoniae strains had solitary abscess $(\mathrm{P}=0.043$ vs ST23 strains). There were no significant differences of liver abscess sizes and complications between ST23 and nonST23 K. pneumoniae isolates (Table 5).

\section{Discussion}

Pyogenic liver abscess is a potentially life-threatening disease. Although Escherichia coli was the most common pathogen in liver abscess before the 1980s, K. pneumoniae had become the dominant pathogen for liver abscess during the past two decades. In Taiwan, over $80 \%$ of bacterial liver abscesses were caused by K. pneumoniae. In Singapore and South Korea, the prevalence of KLA was similar. In our medical center, approximate $81.7 \%$ of pyogenic liver abscess were caused by $K$. pneumoniae. Male gender, patients with diabetes mellitus and biliary disease were more likely to have KLA. Most patients had a single abscess on the right hepatic lobe. The low mortality rate has been similarly observed in recent reports on liver abscess [20,21].

Although liver abscesses caused by extended spectrum $\beta$-lactamase (ESBL)-producing $K$. pneumoniae have been reported, it is a rare occurrence [21]. In our study, only $1 \mathrm{~K}$. pneumoniae strain produced ESBL, which was isolated from a KLA patient who had an accident injury one month ago. As previous reported, K. pneumoniae isolates from KLA were susceptible to almost all kinds of antimicrobial agents such as third generation cephalosporins, $\beta$-lactamase inhibitor compounds, and carbapenems [2,22].

The K1 K. pneumoniae isolates contributed to $68.9 \%$ of KLA in this study, which was higher than previous reports from mainland China (43\% and 39.2\%) [15,20,23]. The prevalence of $\mathrm{K} 1$ isolates was significantly higher than K2 in this study (68.9\% vs 20\%). Our MLST data of the $K$. pneumoniae isolates from KLA patients revealed that ST23 was predominant sequence type with a rate of $57.8 \%$, which was higher than that in the recent report from North China (37.2\%) [15]. This indicated that serotypes and MLST of KLA K. pneumoniae maybe different among various regions in China. Nationwide study would be necessary to realize the national molecular epidemiology of KLA $K$. pneumoniae in China. In our study, serotype K1 KLA $K$. pneumonia isolates that mainly belong to ST23, while ST65-like and ST86-like 
are the two major MLST types among serotype K2 isolates. These results were similar with Siu-LK et al's recent report from Asia [14].

PFGE results demonstrated that there was no clonal dissemination among the 26 ST23 K. pneumoniae isolates. Most of ST23 K. pneumoniae isolates (96.2\%) belonged to K1 serotype with magA positive. As previously reported, ST23 was the most prevalent sequence type among serotype $\mathrm{K} 1 \mathrm{~K}$. pneumoniae isolates from both liver abscess and stool samples in the Asia Pacific region [2,24-26]. It implies that liver abscess might develop after leakage of $K$. pneumoniae from a patient's bowel into their liver via the portal circulation [25-27].

magA has been described as the causative gene for K. pneumoniae liver abscess and septic metastatic complications [7]. The enzyme encoded by magA, also named $w z y$ in accordance with the bacterial polysaccharide gene nomenclature scheme, functions as a polymerase involved in capsule synthesis, and this function is restricted to the capsular gene cluster of serotype K1 only $[10,28]$. In previous study, magA has been reported in $98.1 \%$ and $83.3 \%$ of $K$. pneumoniae strains isolated from patients with liver abscess and was significantly more prevalent than the bacteremic strains $[17,28,29]$. However, our study showed that magA was detected in $68.9 \%$ of K. pneumoniae isolates from KLA patients, and 96.2\% in ST23 K. pneumoniae isolates from KLA patients. Therefore, almost all ST23 K. pneumoniae isolates from KLA patients was magA-positive and K1 serotype, but not all of the magA-positive and K1 serotype $K$. pneumoniae isolates from KLA patients belonged to ST23.

rmpA has been confirmed to regulate capsular polysaccharide synthesis and was proposed as a virulent factor in addition to magA and capsular serotypes $\mathrm{K} 1 / \mathrm{K} 2$ $[8,30]$. Recently, $r m p A$-associated hypermucoviscosity phenotype has also been reported to play an important role in invasive purulent diseases caused by $K$. pneumoniae. K. pneumoniae serotypes $\mathrm{K} 1$ and $\mathrm{K} 2$ isolated from patients with liver abscess usually carrying hypermucoviscosity [2,5]. Our study showed that all $K$. pneumoniae strains that cause liver abscesses were rmpA-positive. However, our string test results revealed that only $28.9 \%$ K. pneumoniae isolates from KLA showed hypermucoviscosity. The hvKP rate was significantly lower than that reported by previous studies (more than $85 \%$ ) and also the recent report from mainland China (70.6\%) $[5,8,15]$. We had repeated the string test for three times and received the same results. It seemed that string test might not be a reliable method to identify $K$. pneumoniae with the potential to cause KLA. All of the $13 \mathrm{~K}$. pneumoniae strains with hypermucoviscosity (hvKP) belonged to K1/ K2 serotype. The prevalence of cKP in K. pneumoniae isolates from diabetes mellitus and drinker patients were higher than that of hvKP. It was consistence with that hvKP was more liable to cause KLA in healthy hosts than cKP [5]. However, there was no significant difference of complications and prognosis between patients infected with hvKP and cKP, ST23 and non-ST23, or magA-positive and -negative strains.

Our study also has several limitations. First, any potential for selection bias in terms of those PLAs aspirated or those patients presenting to this particular institute. Second, that there is no denominator data here; neither in terms of number of PLAs in total, not of number of K. pneumoniae invasive isolates (i.e. positive blood cultures) which did not result in PLA. Third, although there was no significant difference of complications and prognosis among patients infected with different $K$. pneumoniae isolates, the sample size of our study was relative small. In order to determine the predictor of metastatic infection and prognosis of KLA, more KLA cases should be collected for further study.

\section{Conclusions}

In summary, K1 serotype and ST23 were the predominant serotype and sequence type of KLA K. pneumoniae in our study. The hvKP rate was significantly lower than that in previous reports. There was no significant association between the microbiological characteristics including serotypes, magA and $r m p A$ genotypes, and STs with the metastatic infection and prognosis of KLA in this study. Further studies with larger sample are needed in the future.

\section{Additional file}

Additional file 1: Table S1. MLST and serotype analysis in K. pneumoniae

isolates from liver abscess.

\section{Competing interests}

The authors declare that there are no competing interests.

\section{Authors' contributions}

$T T Q$ : conceived of the study, and participated in its design and coordination; JCZ and YJ: analyzed and interpreted the data; KRS and BL: performed the laboratory experiments, reviewed and collected the data; PS and ZQW: acquisition of data, technical support; YSY: study concept and design, analysis and interpretation of data, drafting of the manuscript, study supervision. All authors read and approved the final manuscript.

\section{Acknowledgements}

This work was supported by the research grant from the National Natural Science Foundations of China (No.NSFC81171615, 81301460, and 81401708).

\section{Author details}

${ }^{1}$ State Key Laboratory for Diagnosis and treatment of Infectious Disease, First Affiliated Hospital, College of Medicine, Zhejiang University, 3\# Qingchun East Road, Hangzhou 310016, China. ${ }^{2}$ Department of Infectious Diseases, Sir Run Run Shaw Hospital, College of Medicine, Zhejiang University, Hangzhou, Zhejiang, China. ${ }^{3}$ Department of Infectious Diseases, Forth Affiliated Hospital, College of Medicine, Zhejiang University, Yiwu, Zhejiang, China.

${ }^{4}$ Collaborative Innovation Center for Diagnosis and Treatment of Infectious Diseases, Hangzhou, Zhejiang, China. 
Received: 3 July 2014 Accepted: 13 March 2015

Published online: 27 March 2015

\section{References}

1. Lin YT, Wang FD, Wu PF, Fung CP. Klebsiella pneumoniae liver abscess in diabetic patients: association of glycemic control with the clinical characteristics. BMC Infect Dis. 2013;13:56.

2. Siu LK, Yeh KM, Lin JC, Fung CP, Chang FY. Klebsiella pneumoniae liver abscess: a new invasive syndrome. Lancet Infect Dis. 2012;12(11):881-7.

3. Fang $C T$, Lai SY, Yi WC, Hsueh PR, Liu KL, Chang SC. Klebsiella pneumoniae genotype K1: an emerging pathogen that causes septic ocular or central nervous system complications from pyogenic liver abscess. Clin Infect Dis. 2007:45(3):284-93.

4. Lin YC, Lu MC, Tang HL, Liu HC, Chen CH, Liu KS, et al. Assessment of hypermucoviscosity as a virulence factor for experimental Klebsiella pneumoniae infections: comparative virulence analysis with hypermucoviscosity-negative strain. BMC Microbiol. 2011;11:50.

5. Shon AS, Bajwa RP, Russo TA. Hypervirulent (hypermucoviscous) Klebsiella pneumoniae: a new and dangerous breed. Virulence. 2013;4(2):107-18

6. Yeh KM, Chang FY, Fung CP, Lin JC, Siu LK. magA is not a specific virulence gene for Klebsiella pneumoniae strains causing liver abscess but is part of the capsular polysaccharide gene cluster of K. pneumoniae serotype K1. J Med Microbiol. 2006;55(Pt 6):803-4.

7. Fang CT, Lai SY, Yi WC, Hsueh PR, Liu KL. The function of wzy_K1 (magA), the serotype $\mathrm{K} 1$ polymerase gene in Klebsiella pneumoniae cps gene cluster. J Infect Dis. 2010;201(8):1268-9.

8. Yu WL, Ko WC, Cheng KC, Lee HC, Ke DS, Lee CC, et al. Association between rmpA and magA genes and clinical syndromes caused by Klebsiella pneumoniae in Taiwan. Clin Infect Dis. 2006;42(10):1351-8.

9. Yeh KM, Lin JC, Yin FY, Fung CP, Hung HC, Siu LK, et al. Revisiting the importance of virulence determinant magA and its surrounding genes in Klebsiella pneumoniae causing pyogenic liver abscesses: exact role in serotype K1 capsule formation. J Infect Dis. 2010;201(8):1259-67.

10. Struve C, Bojer M, Nielsen EM, Hansen DS, Krogfelt KA. Investigation of the putative virulence gene magA in a worldwide collection of 495 Klebsiella isolates: magA is restricted to the gene cluster of Klebsiella pneumoniae capsule serotype K1. J Med Microbiol. 2005;54(Pt 11):1111-3.

11. Tang HL, Chiang MK, Liou WJ, Chen YT, Peng HL, Chiou CS, et al. Correlation between Klebsiella pneumoniae carrying pLVPK-derived loci and abscess formation. Eur J Clin Microbiol Infect Dis. 2010;29(6):689-98.

12. Chuang YC, Lee MF, Tan CK, Ko WC, Wang FD, Yu WL. Can the rmpA gene predict metastatic meningitis among patients with primary Klebsiella pneumoniae liver abscess? J Infection. 2013;67(2):166-8.

13. Merlet A, Cazanave C, Dutronc H, de Barbeyrac B, Brisse S, Dupon M. Primary liver abscess due to CC23-K1 virulent clone of Klebsiella pneumoniae in France. Clin Microbiol Infect. 2012;18(9):E338-339.

14. Lin JC, Koh TH, Lee N, Fung CP, Chang FY, Tsai YK, et al. Genotypes and virulence in serotype K2 Klebsiella pneumoniae from liver abscess and noninfectious carriers in Hong Kong, Singapore and Taiwan. Gut Pathogens. 2014;6:21.

15. Luo Y, Wang Y, Ye L, Yang J. Molecular epidemiology and virulence factors of pyogenic liver abscess causing klebsiella pneumoniae in china. Clin Microbiol Infect. 2014;20(11):818-24.

16. Li W, Sun G, Yu Y, Li N, Chen M, Jin R, et al. Increasing occurrence of antimicrobial-resistant hypervirulent (hypermucoviscous) Klebsiella pneumoniae isolates in China. Clin Infect Dis. 2013;58(2):225-32.

17. Yeh KM, Kurup A, Siu LK, Koh YL, Fung CP, Lin JC, et al. Capsular serotype $\mathrm{K} 1$ or $\mathrm{K} 2$, rather than magA and rmpA, is a major virulence determinant for Klebsiella pneumoniae liver abscess in Singapore and Taiwan. J Clin Microbiol. 2007:45(2):466-71.

18. Yang J, Ye L, Guo L, Zhao Q, Chen R, Luo Y, et al. A nosocomial outbreak of KPC-2-producing Klebsiella pneumoniae in a Chinese hospital: dissemination of ST11 and emergence of ST37, ST392 and ST395. Clin Microbiol Infect. 2013;19(11):E509-515.

19. Tenover FC, Arbeit RD, Goering RV, Mickelsen PA, Murray BE, Persing DH, et al. Interpreting chromosomal DNA restriction patterns produced by pulsed-field gel electrophoresis: criteria for bacterial strain typing. J Clin Microbiol. 1995;33(9):2233-9.

20. Chung DR, Lee SS, Lee HR, Kim HB, Choi HJ, Eom JS, et al. Emerging invasive liver abscess caused by $\mathrm{K} 1$ serotype Klebsiella pneumoniae in Korea. J Infection. 2007;54(6):578-83.
21. Rahimian J, Wilson T, Oram V, Holzman RS. Pyogenic liver abscess: recent trends in etiology and mortality. Clin Infect Dis. 2004;39(11):1654-9.

22. Moore R, O'Shea D, Geoghegan T, Mallon PW, Sheehan G. Communityacquired Klebsiella pneumoniae liver abscess: an emerging infection in Ireland and Europe. Infection. 2013;41(3):681-6.

23. Shen DX, Wang J, Li DD. Klebsiella pneumoniae liver abscesses. Lancet Infect Dis. 2013;13(5):390-1.

24. Liao CH, Huang YT, Chang CY, Hsu HS, Hsueh PR. Capsular serotypes and multilocus sequence types of bacteremic Klebsiella pneumoniae isolates associated with different types of infections. Eur J Clin Microbiol Infect Dis. 2014;33(3):365-9.

25. Chung DR, Lee $\mathrm{H}$, Park MH, Jung SI, Chang HH, Kim YS, et al. Fecal carriage of serotype K1 Klebsiella pneumoniae ST23 strains closely related to liver abscess isolates in Koreans living in Korea. Eur J Clin Microbiol Infect Dis. 2012;31(4):481-6.

26. Siu LK, Fung CP, Chang FY, Lee N, Yeh KM, Koh TH, et al. Molecular typing and virulence analysis of serotype K1 Klebsiella pneumoniae strains isolated from liver abscess patients and stool samples from noninfectious subjects in Hong Kong, Singapore, and Taiwan. J Clin Microbiol. 2011;49(11):3761-5.

27. Fung CP, Lin YT, Lin JC, Chen TL, Yeh KM, Chang FY, et al. Klebsiella pneumoniae in gastrointestinal tract and pyogenic liver abscess. Emerg Infect Dis. 2012;18(8):1322-5.

28. Chuang YP, Fang CT, Lai SY, Chang SC, Wang JT. Genetic determinants of capsular serotype $\mathrm{K} 1$ of Klebsiella pneumoniae causing primary pyogenic liver abscess. J Infect Dis. 2006;193(5):645-54.

29. Fung CP, Chang FY, Lee SC, Hu BS, Kuo Bl, Liu CY, et al. A global emerging disease of Klebsiella pneumoniae liver abscess: is serotype $\mathrm{K} 1$ an important factor for complicated endophthalmitis? Gut. 2002;50(3):420-4.

30. Hsu CR, Lin TL, Chen YC, Chou HC, Wang JT. The role of Klebsiella pneumoniae rmpA in capsular polysaccharide synthesis and virulence revisited. Microbiology. 2011;157(Pt 12):3446-57.

\section{Submit your next manuscript to BioMed Central and take full advantage of:}

- Convenient online submission

- Thorough peer review

- No space constraints or color figure charges

- Immediate publication on acceptance

- Inclusion in PubMed, CAS, Scopus and Google Scholar

- Research which is freely available for redistribution 